UT-STPD-4/03

\title{
Leptogenesis through direct inflaton decay to light particles
}

\author{
Thomas Dent $⿴ 囗 十$ George Lazarides 0 and Roberto Ruiz de Austr: \\ Physics Division, School of Technology, Aristotle University of Thessaloniki, Thessaloniki 54124, Greece
}

(Dated: October 29, 2018)

\begin{abstract}
We present a scenario of nonthermal leptogenesis following supersymmetric hybrid inflation, in the case where inflaton decay to both heavy right handed neutrino and $\mathrm{SU}(2)_{L}$ triplet superfields is kinematically disallowed. Lepton asymmetry is generated through the decay of the inflaton into light particles by the interference of one-loop diagrams with right handed neutrino and $\mathrm{SU}(2)_{L}$ triplet exchange respectively. We require superpotential couplings explicitly violating a U(1) Rsymmetry and R-parity. However, the broken R-parity need not have currently observable lowenergy signatures. Also, the lightest sparticle can be stable. Some R-parity violating slepton decays may, though, be detectable in the future colliders. We take into account the constraints from neutrino masses and mixing and the preservation of the primordial lepton asymmetry.
\end{abstract}

PACS numbers: 98.80.Cq, 12.10.Dm, 12.60.Jv

\section{INTRODUCTION}

The standard model (SM) of electroweak and strong interactions, despite its many successes, leaves unanswered many questions in particle physics and cosmology. In particular, it does not address the following problems: the origin of electroweak symmetry breaking and of the hierarchy between the electroweak scale $M_{W}$ and the (reduced) Planck scale $m_{\mathrm{P}} \simeq 2.44 \times 10^{18} \mathrm{GeV}$; the origin and size of neutrino $(\nu)$ masses and mixing; the cosmological horizon and flatness problems and the origin of density perturbations; the generation of the observed baryon asymmetry of the universe (BAU).

The problem of stabilizing the electroweak scale relative to the fundamental scale $M_{S}$ (which we take to be close to the Planck scale) is solved by softly-broken supersymmetry (SUSY). For definiteness, we take the case of gravity-mediated SUSY-breaking, where the gravitino mass is of order $1 \mathrm{TeV}$. In this framework, the vacuum expectation values (VEVs) of the electroweak Higgs superfields $h_{1}, h_{2}$ can be determined by radiative corrections to the scalar potential [1]. However, this explanation of the electroweak symmetry-breaking requires a term $\mu h_{1} h_{2}$ in the superpotential with $\mu \sim 1 \mathrm{TeV}$, whereas a priori the value of $\mu$ is expected to be of the order of the fundamental scale. This $\mu$ problem of the minimal SUSY standard model (MSSM) can be addressed by imposing a symmetry that forbids the above $\mu$ term, which is then broken in a controlled fashion. A R-symmetry, broken by the soft SUSY-breaking terms, can fulfill 22 this role (see below).

The smallness of neutrino masses is naturally explained in a model with additional SM singlet chiral fermions $\left(\nu^{c}\right)$, the right handed neutrinos (RHNs), since these can obtain heavy Majorana masses $\left(\sim M_{\nu^{c}}\right)$ and also par-

\footnotetext{
${ }^{*}$ Electronic address: tdent@gen.auth.gr

${ }^{\dagger}$ Electronic address: lazaride@eng.auth.gr

${ }^{\ddagger}$ Electronic address: rruiz@gen.auth.gr
}

ticipate in Yukawa couplings with the $\mathrm{SU}(2)_{L}$ doublet neutrinos resulting in Dirac neutrino masses $\left(\sim m_{D}\right)$ after the electroweak symmetry breaking. The resulting seesaw mass matrix [3] has small eigenvalues of order $m_{D}^{2} / M_{\nu^{c}}$, which can be of the right order of magnitude to explain atmospheric and solar neutrino oscillations, while being consistent with cosmological bounds on neutrino masses. Another mechanism of inducing nonzero neutrino masses is the introduction of heavy scalar $\mathrm{SU}(2)_{L}$ triplets $T$ with lepton number -2 , coupling to the $\mathrm{SU}(2)_{L}$ doublet lepton fields. After the breaking of the electroweak symmetry, the neutral components of these triplets can acquire small VEVs inducing Majorana neutrino masses [4]. In general, these two mechanisms for generating neutrino masses can coexist.

The cosmological horizon and flatness problems are most elegantly solved by inflation, which can also generate the primordial density perturbations required for structure formation in the universe [5]. Moreover, inflation, which can be easily incorporated in realistic particle physics models, is strongly favored by the recent data $[6$ on the angular power spectrum of the cosmic microwave background radiation (CMBR).

If neutrinos get mass either by coupling to heavy SM singlet fermions or to heavy scalar $\mathrm{SU}(2)_{L}$ triplets, it may be possible to generate [7, 8] a primordial lepton asymmetry in the out-of-equilibrium decay of these heavy degrees of freedom, in case they were thermally produced in the early universe. This asymmetry is then reprocessed at the electroweak phase transition to yield the observed BAU. However, there is a tension between correct neutrino masses and this thermal leptogenesis scenario in SUSY models because of the gravitino problem [9, 10]. With a gravitino mass of around $1 \mathrm{TeV}$, the reheat temperature $T_{\text {rh }}$ should not exceed $10^{9} \mathrm{GeV}$ since gravitinos produced in thermal scattering processes would decay late presumably into photons and photinos and, if sufficiently numerous, interfere with the successful predictions of standard big bang nucleosynthesis. On the other hand, one also requires that the heavy fields whose decay creates lepton asymmetry be generated in sufficient 
numbers. So, their masses should not exceed $T_{\mathrm{rh}}$, which leads to unacceptably large light neutrino masses. However, this problem can be alleviated [11, 12] by assuming that there is some degree of degeneracy between the relevant RHNs or heavy $\mathrm{SU}(2)_{L}$ triplets, which enhances the generated lepton asymmetry, and perhaps also that the branching ratio of the gravitino decay into photons and photinos is less than unity, which somewhat relaxes [10] the gravitino constraint on $T_{\mathrm{rh}}$.

The tension between $\nu$ masses and the gravitino problem can be more naturally relaxed by considering nonthermal leptogenesis 13 at reheating. However, in existing scenarios [14, 15], where the inflaton decays into RHN or $\mathrm{SU}(2)_{L}$ triplet superfields, this still puts a restriction on the masses of these particles: the decay products of the inflaton must be lighter than half its mass $m_{\text {inf }}$. Lepton asymmetry is generated in the subsequent decay of the RHN or $\mathrm{SU}(2)_{L}$ triplet superfields.

In this work, however, we consider the consequences of allowing all the $\mathrm{RHN}$ and/or $\mathrm{SU}(2)_{L}$ triplet superfields of the model to be heavier than $m_{\text {inf }} / 2$ (see also Ref. [16]). Leptogenesis could then occur only via the direct decay of the inflaton to light particles (see also Ref. [17]). We take a simple SUSY grand unified theory (GUT) model naturally incorporating the standard SUSY realization [18, 19] of hybrid inflation [20], which does not require tiny parameters and is, undoubtedly, one of the most promising inflationary scenarios. (For extensions of standard SUSY hybrid inflation, see Ref. [21].) In global SUSY, the flatness of the inflationary path at tree level is guaranteed by a U(1) R-symmetry. The $\eta$ problem [18] of sizable supergravity (SUGRA) contributions to the inflaton mass on the inflationary path, which could easily invalidate inflation, is reduced, in this case, to controlling the magnitude of a single term in the Kähler potential 22] (see also Ref. 23]). Finally, radiative corrections provide [19] a logarithmic slope along the classically flat direction, needed for driving the inflaton towards the SUSY vacua.

The $\mu$ problem is solved by employing the mechanism of Ref. 22]. (For an alternative solution of the $\mu$ problem, see Ref. [14].) The global R-symmetry of the model forbids the appearance of a $\mu$ term in the superpotential. On the contrary, it allows the existence of the trilinear term $S h_{1} h_{2}$, where $S$ is the gauge singlet inflaton of the standard SUSY hybrid inflation. After the GUT gauge symmetry breaking, the soft SUSY-breaking terms, which generally violate the R-symmetry, give rise to a suppressed linear term in $S$ and, thus, this field acquires a VEV of order the electroweak scale divided by a small coupling constant. The above trilinear coupling can then yield a $\mu$ term of the right magnitude.

The inflaton consists of two complex scalar fields with tree-level couplings to the electroweak Higgses and Higgsinos derived from the above trilinear term. After the end of inflation, it oscillates about the SUSY vacuum and eventually decays predominantly into electroweak Higgs superfields via these tree-level couplings, thereby reheat- ing the universe. We find that, in the case of one fermion family, both heavy RHN and $\mathrm{SU}(2)_{L}$ triplet superfields are necessary if diagrams producing a nonzero lepton asymmetry are to exist. Since these heavy fields can only appear in intermediate states of the inflaton decay, we must create the asymmetry directly from this decay. Indeed, leptogenesis can occur in the subdominant decay of the inflaton into lepton and Higgs superfields through the interference between different one-loop diagrams with RHN and $\mathrm{SU}(2)_{L}$ triplet exchange respectively. The lepton asymmetry is proportional to a novel CP-violating invariant product of coupling constants.

For a nonzero asymmetry, we also need to include some couplings in the superpotential that explicitly violate both the $\mathrm{U}(1) \mathrm{R}$-symmetry and its $Z_{2}$ matter parity subgroup, which remains unbroken by the soft SUSYbreaking terms. Although these couplings involve superheavy fields, the matter parity violation may have some observable consequences at low energy such as the possible instability of the lightest SUSY particle (LSP). Indeed, if this particle contains a Higgsino component, it could decay predominantly into a pair of Higgses and a lepton. This channel can, though, be easily blocked kinematically if the LSP is not too heavy (see Sec. D). On the contrary, some R-parity violating slepton decays which may be detectable in the future colliders are typically present (see Sec. VIII).

We find that the value of the BAU from the Wilkinson microwave anisotropy probe (WMAP) data [6] can be easily achieved given constraints from other observables, notably the reheat temperature and neutrino masses and mixing, and CP-violating phases of order unity. However, the constraint from $\nu$ masses and mixing is considerably weakened by the existence of two separate contributions, namely those of the usual seesaw mechanism and from the Higgs $\mathrm{SU}(2)_{L}$ triplets (see alsoc Ref. 24]). On the contrary, the requirement that the initial lepton asymmetry is protected from lepton number violating $2 \rightarrow 2$ scattering processes which are in equilibrium in the early universe imposes very stringent constraints on the parameters of the model, which we also take into account in our analysis. The prediction for the spectral index of density perturbations is typical of SUSY hybrid inflation models (see e.g. Ref. [25]).

Thus, an acceptable value of the BAU can be obtained within a consistent model of cosmology and particle physics, without requiring additional fine-tuned coupling constants and without necessarily putting strong constraints on observables such as neutrino masses and mixing. Moreover, although the scenario requires violation of the R-symmetry, it is not necessary to introduce superpotential terms which would lead to currently observable R-symmetry violating effects.

In Sec. III we introduce our model and describe some of its salient features. In Sec. IIII we present the CPviolating invariant products of coupling constants which enter into the primordial lepton asymmetry, while, in Sec. IV] we sketch the calculation of the BAU. The ef- 
TABLE I: U(1) charges of superfields

\begin{tabular}{c|ccccccccccccc}
\hline \hline & $S$ & $\phi$ & $\bar{\phi}$ & $T$ & $\bar{T}$ & $h_{1}$ & $h_{2}$ & $l$ & $\nu^{c}$ & $e^{c}$ & $q$ & $u^{c}$ & $d^{c}$ \\
\hline$B-L$ & 0 & 1 & -1 & 2 & 0 & 0 & 0 & -1 & 1 & 1 & $1 / 3$ & $-1 / 3$ & $-1 / 3$ \\
$R$ & 2 & 0 & 0 & 0 & 2 & 0 & 0 & 1 & 1 & 1 & 1 & 1 & 1 \\
\hline \hline
\end{tabular}

fects of R-symmetry violation are discussed in Sec. $\mathbb{V}$ The constraints from neutrino masses and the preservation of the initial lepton asymmetry are given in Sec. VI] and our numerical results in Sec. VII Finally, our conclusions are summarized in Sec. VIII

\section{THE MODEL}

The model has gauge group $\mathrm{SU}(3)_{c} \times \mathrm{SU}(2)_{L} \times \mathrm{U}(1)_{Y} \times$ $\mathrm{U}(1)_{B-L}$ and a global R-symmetry $\mathrm{U}(1)_{R}$, which is though explicitly broken by some terms in the superpotential (see below). In addition to the corresponding vector superfields and the usual MSSM chiral superfields $h_{1}$, $h_{2}$ (Higgs $\mathrm{SU}(2)_{L}$ doublets), $l_{i}\left(\mathrm{SU}(2)_{L}\right.$ doublet leptons), $e_{i}^{c}\left(\mathrm{SU}(2)_{L}\right.$ singlet charged leptons $), q_{i}\left(\mathrm{SU}(2)_{L}\right.$ doublet quarks), $u_{i}^{c}, d_{i}^{c}\left(\mathrm{SU}(2)_{L}\right.$ singlet quarks) with $i=1,2,3$ being the family index, we introduce chiral superfields $\nu_{i}^{c}$ (RHNs), $S, \phi, \bar{\phi}$ singlets under the SM gauge group and $T, \bar{T}$ in the adjoint representation of $\mathrm{SU}(2)_{L}$ with $Y=1,-1$ respectively. (As usual, we will use the same symbol to denote the superfield and its scalar component, the distinction being clear in context.) The charges under $\mathrm{U}(1)_{B-L}$ and $\mathrm{U}(1)_{R}$ are given in Table I.

The superpotential is

$$
\begin{aligned}
W & =\kappa S\left(\phi \bar{\phi}-M^{2}\right)+\lambda S\left(h_{1} h_{2}\right) \\
& +h_{e i j}\left(h_{1} l_{i}\right) e_{j}^{c}+h_{u i j}\left(h_{2} q_{i}\right) u_{j}^{c}+h_{d i j}\left(h_{1} q_{i}\right) d_{j}^{c} \\
& +h_{\nu i j}\left(h_{2} l_{i}\right) \nu_{j}^{c}+h_{T i j} l_{i} \epsilon T l_{j}+h_{\bar{T}} h_{2} \epsilon \bar{T} h_{2} \\
& +\left(M_{\nu^{c} i j} / M^{2}\right) \bar{\phi}^{2} \nu_{i}^{c} \nu_{j}^{c}+\left(M_{T} / M^{2}\right) \bar{\phi}^{2} T \bar{T} \\
& +\left(\lambda_{i} / M_{S}\right) \bar{\phi}\left(h_{1} h_{2}\right) \nu_{i}^{c}+\left(\lambda_{i}^{\prime} / M_{S}\right) \bar{\phi} h_{1} \epsilon T l_{i}+\cdots,
\end{aligned}
$$

where $M$ is a mass parameter of the order of the GUT scale, $M_{S}$ is the string scale $\simeq 5 \times 10^{17} \mathrm{GeV}, \epsilon$ is the $2 \times 2$ antisymmetric matrix with $\epsilon_{12}=1,(X Y)$ indicates the $\mathrm{SU}(2)_{L}$ invariant product $\epsilon_{a b} X_{a} Y_{b}$ and $T \equiv T_{a} \sigma_{a} / \sqrt{2}$, $\bar{T} \equiv \bar{T}_{a} \sigma_{a} / \sqrt{2}$ with $\sigma_{a}(a=1,2,3)$ being the Pauli matrices. The ellipsis represents terms of order higher than four and summation over indices is implied. The only $\mathrm{U}(1)_{R}$ violating terms which we allow in the superpotential are the two explicitly displayed terms in the last line of the right hand side (RHS) of Eq. (1), which are necessary for leptogenesis. We can show that baryon number $(B)$ is automatically conserved to all orders as a consequence of $\mathrm{U}(1)_{R}$. The argument goes as in Ref. 15] and is not affected by the presence of the above $\mathrm{U}(1)_{R}$ breaking superpotential terms. Lepton number $(L)$ is then also conserved as implied by the presence of $\mathrm{U}(1)_{B-L}$.

The inflationary trajectory is as described in Ref. [2]: for $\kappa<\lambda$, it is parametrized by $S,|S|>S_{c}=M$, with all the other fields vanishing and has a constant energy density $\kappa^{2} M^{4}$ at tree level. Here, the dimensionless parameters $\kappa, \lambda$ and the mass $M$ are taken real and positive by redefining the phases of the superfields. There are radiative corrections [19] which lift the flatness of this classically flat direction leading to slow-roll inflation until $|S|$ reaches the instability point at $|S|=M$ as one can deduce from the $\epsilon$ and $\eta$ criteria [25]. The quadrupole anisotropy of the CMBR and the number of e-foldings $N_{Q} \simeq \ln \left[1.88 \times 10^{11} \kappa^{1 / 3}(M / \mathrm{GeV})^{2 / 3}\left(T_{\mathrm{rh}} / \mathrm{GeV}\right)^{1 / 3}\right][25]$ of our present horizon scale during inflation are given by the Eqs. (2)-(4) of Ref. [26] with the two last terms in the RHS of Eq. (3) divided by two since the $\mathrm{SU}(2)_{R}$ doublet chiral superfields $l^{c}, \bar{l}^{c}$ of this reference are now replaced by the SM singlets $\phi, \bar{\phi}$ (see also Ref. [27]).

When the value of $|S|$ falls below $M$, a $B-L$ breaking phase transition occurs provided that $\kappa<\lambda$. The fields evolve towards the realistic SUSY minimum at $\langle S\rangle=0$, $\langle\phi\rangle=\langle\bar{\phi}\rangle=M,\left\langle h_{1}\right\rangle=\left\langle h_{2}\right\rangle=0$, where $\langle\phi\rangle,\langle\bar{\phi}\rangle$ are taken real and positive by a $B-L$ rotation (there is also an unrealistic SUSY minimum which is given below). Actually, with the addition of soft SUSY-breaking terms, the position of the vacuum shifts [2] to nonzero $\langle S\rangle \simeq-m_{3 / 2} / \kappa$, where $m_{3 / 2}$ is the gravitino mass, and a small effective $\mu$ term with $\mu \simeq-\lambda m_{3 / 2} / \kappa$ is generated from the superpotential coupling $\lambda S\left(h_{1} h_{2}\right)$. Subsequently, the inflaton degrees of freedom $S$ and $\theta \equiv(\delta \phi+\delta \bar{\phi}) / \sqrt{2}(\delta \phi=\phi-M$, $\delta \bar{\phi}=\bar{\phi}-M)$ with mass $m_{\mathrm{inf}}=\sqrt{2} \kappa M$ oscillate about this minimum and decay to MSSM degrees of freedom reheating the universe. The predominant decay channels of $S$ and $\theta$ are to fermionic and bosonic $h_{1}, h_{2}$ respectively via tree-level couplings derived from the superpotential terms $\lambda S\left(h_{1} h_{2}\right)$ and $\kappa S \phi \bar{\phi}$. Note that, if $\kappa>\lambda$, the system would end up in the unrealistic SUSY minimum at $\phi=\bar{\phi}=0,\left|h_{1}\right|=\left|h_{2}\right| \simeq(\kappa / \lambda)^{1 / 2} M$, which is degenerate with the realistic one (up to $m_{3 / 2}^{4}$ ) and is separated from it by a potential barrier of order $m_{3 / 2}^{2} M^{2}$.

The RHNs and the $\mathrm{SU}(2)_{L}$ triplets acquire masses $M_{\nu^{c} i j}$ and $M_{T}$ respectively after the spontaneous breaking of $\mathrm{U}(1)_{B-L}$ by $\langle\phi\rangle,\langle\bar{\phi}\rangle$. The terms which appear in the fourth line of the RHS of Eq. (11) can also be written in the form $\lambda_{\nu^{c} i j} \bar{\phi}^{2} \nu_{i}^{c} \nu_{j}^{c} / M_{S}, \lambda_{T} \bar{\phi}^{2} T \bar{T} / M_{S}$ making it clear that the RHN and $\mathrm{SU}(2)_{L}$ triplet masses are suppressed by a factor $M / M_{S}$ relative to $M$. It is possible to redefine superfields to obtain effective mass terms $M_{\nu_{i}^{c}} \nu_{i}^{c} \nu_{i}^{c}$ (which are diagonal in the flavor space) and $M_{T} T \bar{T}$ with $M_{\nu_{i}^{c}}$ and $M_{T}$ real and positive.

Similarly, after the $\mathrm{U}(1)_{B-L}$ breaking, the explicitly displayed terms in the last line of the RHS of Eq. (1) which violate $\mathrm{U}(1)_{R}$ give rise to effective $B-L$ and matter parity violating operators $\zeta_{i}\left(h_{1} h_{2}\right) \nu_{i}^{c}$ and $\zeta_{i}^{\prime} h_{1} \epsilon T l_{i}$, where $\zeta_{i}$ and $\zeta_{i}^{\prime}$ are suppressed by one power of $M / M_{S}$. If we require that the magnitude of the dimensionless coupling constants $\lambda_{i}$ and $\lambda_{i}^{\prime}$ is less than unity, we obtain the bound $\left|\zeta_{i}\right|,\left|\zeta_{i}^{\prime}\right| \leq M / M_{S}$. Note that, although $\zeta_{i}^{\prime}$ can be made real and positive by redefining the phase of $l_{i}$, there is no phase freedom left which can do the same 
for $\zeta_{i}$. This can be shown by considering the rephasing invariant $\zeta_{i}^{* 2} \mu^{2} M_{\nu_{i}^{c}}$ (no summation over $i$ ) with $\mu$ and $M_{\nu_{i}^{c}}$ already made real. The coupling constants $\zeta_{i}$, thus, remain in general complex. It is, of course, possible to write down many other R-symmetry violating operators. However, they are not necessary for a nonzero lepton asymmetry to be created.

After electroweak symmetry breaking, the triplet $T$ also acquires [4, 14] a VEV $\langle T\rangle=-h_{\bar{T}}\left\langle h_{2}\right\rangle^{2} / M_{T}$. This is due to the mass term $M_{T} T \bar{T}$ and the coupling $h_{\bar{T}} h_{2} \epsilon \bar{T} h_{2}$, where $h_{\bar{T}}$ is made real and positive by a redefinition of the phase of $\bar{T}$ and a compensating rephasing of $T$ and $l_{i}$ in order to retain the positivity of $M_{T}$ and $\zeta_{i}^{\prime}$. Note that $\left\langle h_{1}\right\rangle,\left\langle h_{2}\right\rangle$ can be taken real because of the reality of $B \mu \simeq-2 \lambda m_{3 / 2}^{2} / \kappa[2]$. So $\langle T\rangle$ is also real.

Performing appropriate flavor rotations of the $l_{i}$ and $e_{j}^{c}$, the Yukawa coupling constant matrix $h_{e i j}$ can be diagonalized with real and positive entries $h_{e i}$ in the diagonal. The neutrino components $\left(\nu_{i}\right)$ of $l_{i}$ are then in the weak interaction basis. (The rephasing of $l_{i}$ which was used to make $\zeta_{i}^{\prime}$ real and positive should actually be performed after these rotations and should be accompanied by a compensating rephasing of $e_{i}^{c}$ so that $h_{e i}$ remains real and positive.) The coupling of $T$ to a pair of lepton $\mathrm{SU}(2)_{L}$ doublets cannot be made diagonal since no further flavor rotations of the $l_{i}$ are allowed. Moreover, if we define superfields such that $M_{T}, \zeta_{i}^{\prime}, \mu$ and $h_{\bar{T}}$ are real then it is in general not possible to make the $h_{T i j}$ real since, once $S, \phi, \bar{\phi}$ acquire nonzero VEVs, we have rephasing invariants $M_{T} h_{T i j} h_{\bar{T}}^{*} \mu^{2} \zeta_{i}^{\prime *} \zeta_{j}^{\prime *}$ (no summation). Finally, the Yukawa coupling constants $h_{\nu i j}$ also remain in general complex as one can easily deduce from the rephasing invariants $h_{\bar{T}}^{* 2} \zeta_{i}^{\prime * 2} M_{T}^{2} \mu^{2} M_{\nu_{j}^{c}}^{*} h_{\nu i j}^{2}$ (no summation over indices).

The calculation of lepton asymmetry produced in $S$ and $\theta$ decays is quite straightforward but somewhat lengthy, and differs in detail from the usual case where leptogenesis occurs via the decay of RHN and/or SU(2) $L$ triplet superfields. Since we consider the interference of two one-loop diagrams, we will need to calculate the real parts of loop integrals which require renormalization.

\section{CP-VIOLATING INVARIANTS}

To produce a nonvanishing net $B-L$ asymmetry from inflaton decay, the theory must contain one or more physical CP-violating quantities. These are products of coupling constants, corresponding to operators noninvariant under CP conjugation, which are nonreal and are not affected by the redefinition of fields by complex phases (or other global symmetries). In this case, we consider the terms of $W$ in Eq. (11). Since leptogenesis takes place at reheating, we work in the vacuum where $\langle\phi\rangle=\langle\bar{\phi}\rangle=M$. For simplicity, we ignore the couplings of the inflaton to RHNs and SU $(2)_{L}$ triplets from the terms in the fourth line of the RHS of Eq. (11) together with its couplings resulting from the two subsequent terms. The inclusion of these couplings would only complicate the analysis without altering the character of our mechanism. So these four superpotential terms are replaced by the effective mass terms $M_{\nu_{i}^{c}} \nu_{i}^{c} \nu_{i}^{c}, M_{T} T \bar{T}$ and couplings $\zeta_{i}\left(h_{1} h_{2}\right) \nu_{i}^{c}$ and $\zeta_{i}^{\prime} h_{1} \epsilon T l_{i}$. The condition of CP invariance is simply that it should be possible to make all products of coupling constants real by field redefinitions.

We consider here only rephasing invariants which, in contrast to the standard invariant used in Ref. [7], would exist even if there was a single fermion family. We find the following independent invariants (no summation over repeated indices):

$$
\begin{aligned}
I_{1 i j} & =M_{T} h_{\nu i j} \zeta_{j}\left(M_{\nu_{j}^{c}} h_{\bar{T}} \zeta_{i}^{\prime}\right)^{*}, \\
I_{2 i j k} & =M_{T} h_{\nu i j} h_{\nu k j}\left(M_{\nu_{j}^{c}} h_{T i k} h_{\bar{T}}\right)^{*},
\end{aligned}
$$

whose imaginary parts violate CP invariance. Any combination of the two (modulo real invariants) results in a third invariant such as $I_{3 i j k}=\zeta_{i}^{\prime} h_{\nu k j}\left(\zeta_{j} h_{T i k}\right)^{*}$. In each case, the expression involves both the $\mathrm{SU}(2)_{L}$ triplet and RHN couplings, thus the generation of a $B-L$ asymmetry is independent of the sources of $\mathrm{CP}$ violation considered in previous scenarios and we require novel decay diagrams. The invariants $I_{1 i j}, I_{2 i j k}, I_{3 i j k}$ are the minimal ones in the sense that they have the smallest possible number of trilinear superpotential couplings.

It is important to note that the invariant $I_{1 i j}$ can be split in two parts $\zeta_{i}^{\prime *} M_{T} h_{\bar{T}}^{*}$ and $\zeta_{j} M_{\nu_{j}^{c}}^{*} h_{\nu i j}$ corresponding to effective operators which carry (opposite) nonzero $B-L$ charges and involve only light fields since the heavy ones can be contracted. These light fields include bosonic or fermionic $h_{1}, h_{2}$ and their conjugates. So, $I_{1 i j}$ is, in principle, suitable for leptogenesis which requires the interference of two $B-L$ violating diagrams. (Recall that the inflaton field couples at tree level to the electroweak Higgs superfields $h_{1}, h_{2}$ and can decay only to light particles.) This is not the case with the rephasing invariant $I_{2 i j k}$ since there are no $h_{1}$ 's among the light fields of the corresponding (effective) operator. On the contrary, the invariant $I_{3 i j k}$ has all the above good properties of $I_{1 i j}$, but one of the interfering amplitudes turns out to be zero in this case. The reason is that it involves a real (with vanishing absorptive part) on-massshell off-diagonal self-energy between $l$ and $h_{1}$ which, in the on-shell (OS) renormalization scheme, vanishes (see Sec.IV]. It is clear that any invariant which can be useful for leptogenesis must involve $I_{1 i j}$ and, thus, the effective coupling constants $\zeta_{j}, \zeta_{i}^{\prime}$. So, the explicit violation of matter parity is essential for our scheme. This is another novel feature of this leptogenesis scenario.

\section{BARYON ASYMMETRY}

The CP-violating rephasing invariant $I_{1 i j}$ corresponds to the product of coupling constants in the interference of the diagrams in Fig. 17 (Fig. 2a) with the diagrams in Figs. 1b and 1, (Figs. 20 and 25) for the $L$ violating 

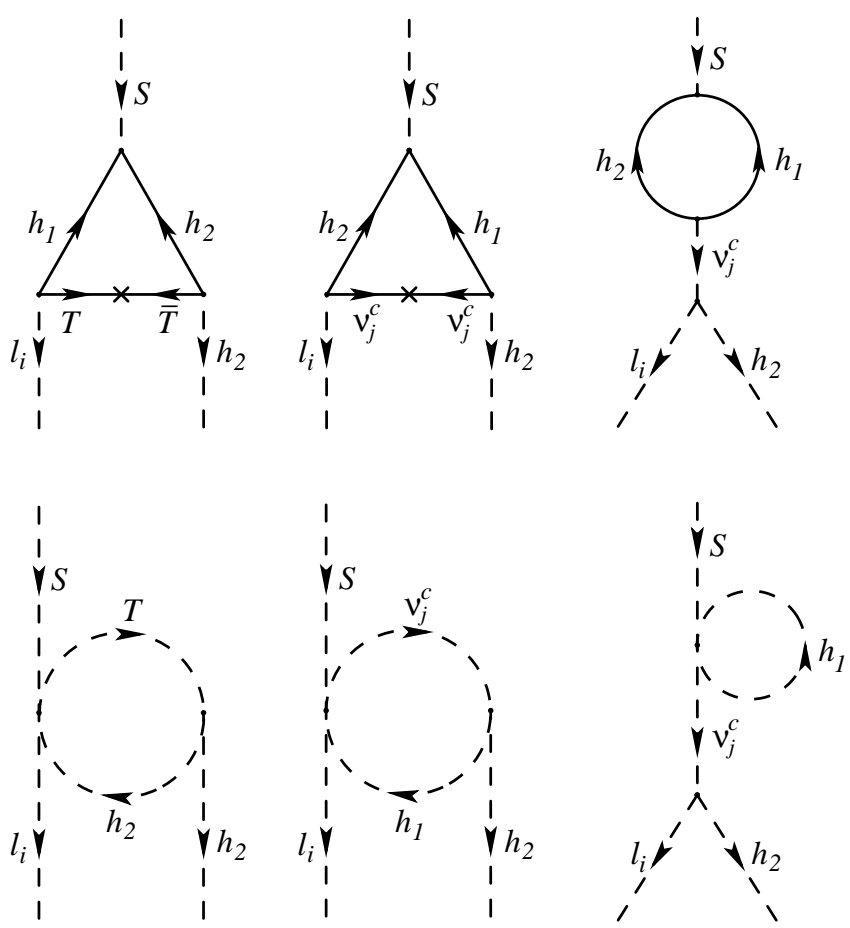

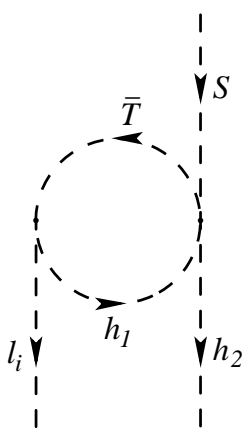

(a)

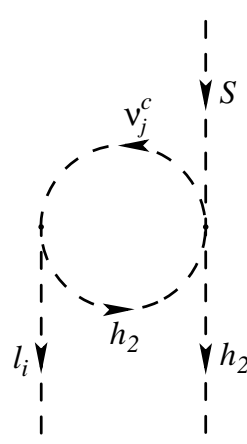

(b)

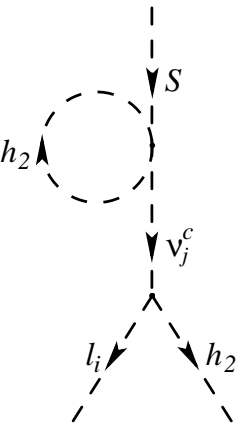

(c)
FIG. 1: The nine one-loop diagrams for the $L$ violating decay $S \rightarrow \tilde{l}_{i} h_{2}$. The solid (dashed) lines represent the fermionic (bosonic) component of the indicated superfield. The arrows depict the chirality of the superfields and the crosses are mass insertions in fermion lines.

decay of $S(\theta)$. This interference contributes to the $L$ asymmetry due to a partial rate difference in the decays $S \rightarrow \tilde{l}_{i} h_{2}$ and $S^{*} \rightarrow \tilde{l}_{i}^{*} h_{2}^{*}\left(\theta^{*} \rightarrow l_{i} \tilde{h}_{2}\right.$ and $\left.\theta \rightarrow \bar{l}_{i} \overline{\tilde{h}}_{2}\right)$, where bar and tilde represent the antifermion and the SUSY partner respectively. Both the decaying inflaton $(S$ or $\theta)$, which we take at rest, and the decay products must be on mass shell. For simplicity, we consider that all the propagating and external MSSM particles in the diagrams are massless. Also, we perform the calculation in the limit of exact SUSY.

In each case, the resulting contribution to the $L$ asymmetry is proportional to both $\operatorname{Im} I_{1 i j}$ and the imaginary part of the interference of the relevant 'stripped' diagrams with the dimensionless coupling constants and the

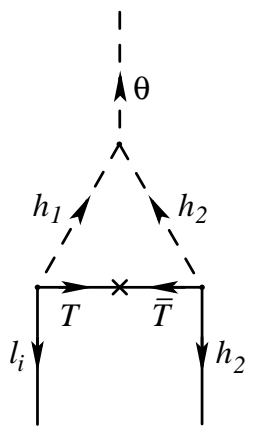

(a)

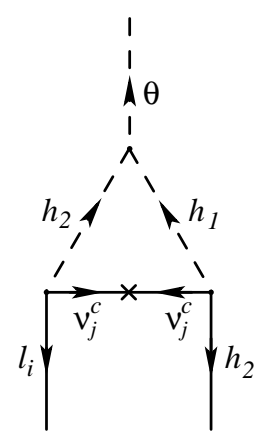

(b)

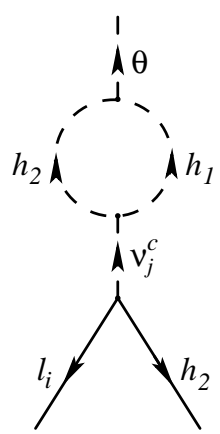

(c)
FIG. 2: The three one-loop diagrams for the $L$ violating decay $\theta^{*} \rightarrow l_{i} \tilde{h}_{2}$. The notation is as in Fig. 1]

$M_{T}, M_{\nu_{j}^{c}}$ mass insertions factored out (we keep, though, the $m_{\text {inf }}$ factor appearing in the scalar coupling $\theta^{*} h_{1} h_{2}$ ). The 'stripped' diagrams in Figs. 1h, 10 and 10 are denoted by $F_{\text {ain }}^{S}, F_{\text {bijn }}^{S}$ and $F_{c i j n}^{S}$ respectively with $i$ and $j$ being the family indices and $n=1,2,3$ the serial number of the diagram. Similarly, the 'stripped' diagrams in Figs. 20 , 20 and 2r are $F_{a i}^{\theta}, F_{b i j}^{\theta}$ and $F_{c i j}^{\theta}$. Thus, the total net $L$ asymmetries $\epsilon_{\mid S}$ and $\epsilon_{\mid \theta}$ generated per $S$ and $\theta$ decay respectively are given by

$$
\begin{aligned}
\epsilon_{\mid S} & =-2 \frac{|\lambda|^{2}}{\Gamma} \operatorname{Im} I_{1 i j} \operatorname{Im}\left[F_{a i n}^{S}{F_{b i j n}^{S}}^{*}+F_{a i n}^{S} F_{c i j n}^{S}{ }^{*}\right], \\
\epsilon_{\mid \theta} & =-2 \frac{|\lambda|^{2}}{\Gamma} \operatorname{Im} I_{1 i j} \operatorname{Im}\left[F_{a i}^{\theta}{F_{b i j}^{\theta}}^{*}+F_{a i}^{\theta} F_{c i j}^{\theta}{ }^{*}\right],
\end{aligned}
$$

where $\Gamma=|\lambda|^{2} m_{\mathrm{inf}} / 8 \pi$ is the rate of the tree-level decays $S \rightarrow \overline{\tilde{h}}_{1} \overline{\tilde{h}}_{2}$ and $\theta \rightarrow h_{1} h_{2}$, and summation over the indices $i, j, n$ and integration over the phase space of the final particles is implied.

We see that the one-loop diagrams for the $L$ violating decay of the inflaton which contain $\mathrm{SU}(2)_{L}$ triplet exchange are exclusively of the vertex type (see Figs. [1, and 22a). On the contrary, the diagrams with a RHN exchange are both of the vertex (Figs. 10 and 2b) and self-energy (Figs. 11 and 2r) 28] type. Each of the three vertex diagrams in Fig. 10 (Fig. 10) possesses a logarithmic ultraviolet (UV) divergence. However, one can easily show that their sum equals $m_{\text {inf }}$ times the vertex diagram in Fig. 22 (Fig. 2b), which is UV finite. Similarly, one can show that, although each of the three self-energy diagrams in Fig. 15 has a quadratic divergence, their sum equals the self-energy diagram in Fig. 2r multiplied by $m_{\text {inf }}$. However, the latter is not UV finite. It rather possesses a logarithmic divergence and, thus, needs renormalization (see below).

The above relations between the diagrams for the $L$ violating decays of $S$ and $\theta$ imply that $\epsilon_{\mid S}=\epsilon_{\mid \theta} \equiv \epsilon$. We can, thus, concentrate on the calculation of $\epsilon_{\mid \theta}$ which is simpler. As already mentioned, the vertex diagrams in Figs. 2 2 and 20 are finite (both their real and imaginary parts) and, thus, well-defined and independent of 
the renormalization scheme used. However, the diagram in Fig. 2: involving a divergent self-energy loop requires us to apply a renormalization condition. As shown in Ref. [11], the appropriate renormalization scheme, in this case, is the on-shell (OS) scheme. In a general theory with scalars $S_{i}$, the OS conditions on the renormalized self-energies $\hat{\Pi}_{i j}\left(p^{2}\right)$ are as follows:

$$
\operatorname{Re} \hat{\Pi}_{i j}\left(\mu_{i}^{2}\right)=\operatorname{Re} \hat{\Pi}_{i j}\left(\mu_{j}^{2}\right)=0
$$

for the off-diagonal self-energies $(i \neq j)$ and

$$
\lim _{p^{2} \rightarrow \mu_{i}^{2}} \frac{1}{p^{2}-\mu_{i}^{2}} \operatorname{Re} \hat{\Pi}_{i i}\left(p^{2}\right)=0
$$

for the diagonal ones (see e.g. Ref. [29]). Here we take a basis where the renormalized mass matrix is diagonal with eigenvalues $\mu_{i}$. The imaginary part of the selfenergies is finite and, thus, not renormalized. In Fig. 2r, we have an off-diagonal self-energy diagram between the scalars $\theta$ and $\tilde{\nu}_{j}^{c}$. Given that $\theta$ is on mass shell, the real part of this diagram vanishes in the OS scheme. The imaginary part, however, gives a finite contribution.

The lepton asymmetry per inflaton decay is calculated by making use of the software packages of Ref. [30] and is found to be given by

$$
\epsilon=\epsilon_{V V}+\epsilon_{V S}
$$

where

$$
\epsilon_{V V}=\frac{3}{128 \pi^{4}} \frac{\operatorname{Im} I_{1 i j}}{m_{\mathrm{inf}}^{2}} \operatorname{Im}\left[f\left(y_{T}\right) f\left(y_{j}\right)^{*}\right]
$$

is the contribution from the interference of the two vertex diagrams in Figs. 2a and 20, and

$$
\epsilon_{V S}=-\frac{3}{64 \pi^{3}} \frac{\operatorname{Im} I_{1 i j}}{m_{\mathrm{inf}}^{2}-M_{\nu_{j}^{c}}^{2}} \operatorname{Re} f\left(y_{T}\right)
$$

is the contribution from the interference of the vertex and self-energy diagrams in Figs. 2 27 and 2 with $f(y)=\pi^{2} / 6-\mathrm{Li}_{2}(1+y+i \varepsilon)\left(\mathrm{Li}_{2}\right.$ is the dilogarithm [31]), $y_{T}=m_{\mathrm{inf}}^{2} / M_{T}^{2}, y_{j}=m_{\mathrm{inf}}^{2} / M_{\nu_{j}^{c}}^{2}$ and summation over the family indices $i, j$ implied. It should be emphasized that Eq. (8) holds [11] provided that the decay width of $\nu_{j}^{c}$ is $\ll\left|m_{\text {inf }}^{2}-M_{\nu_{j}^{c}}^{2}\right| / m_{\text {inf }}$, which is well satisfied in our model if $M_{\nu_{j}^{c}}$ is not unnaturally close to $m_{\text {inf }}$.

The equilibrium conditions including nonperturbative electroweak reactions above the critical temperature of the electroweak phase transition yield a relation between the baryon number density $n_{B}$ and the $B-L$ number density $n_{B-L}$, which allows us to find $n_{B}$ in terms of the $n_{B-L}$ produced from inflaton decay, assuming that $B-L$ is conserved at temperatures well below $M_{T}$ and $M_{\nu_{i}^{c}}$ (see Sec. VI). In the MSSM with soft SUSY-breaking terms, we have $n_{B} / s=(28 / 79) n_{B-L} / s[32$. If we imagine the inflaton to decay instantaneously out of equilibrium creating initial lepton number density $n_{L \text {,init }}$ then

$$
\frac{n_{B}}{s}=-\frac{28}{79} \frac{n_{L, \text { init }}}{s}=-\frac{28}{79} \epsilon \frac{n_{\mathrm{inf}}}{s}=-\frac{21}{79} \epsilon \frac{T_{\mathrm{rh}}}{m_{\mathrm{inf}}}
$$

using the standard relation $n_{\text {inf }} / s \equiv\left(n_{S}+n_{\theta}\right) / s=$ $3 T_{\mathrm{rh}} / 4 m_{\text {inf }}$ for the inflaton number density. Here $s$ is the entropy density. The reheat temperature is given by

$$
T_{\mathrm{rh}}=\left(\frac{45}{2 \pi^{2} g_{*}}\right)^{\frac{1}{4}}\left(\Gamma m_{\mathrm{P}}\right)^{\frac{1}{2}}
$$

where $g_{*}$ counts the relativistic degrees of freedom taking account of the spin and statistics and is equal to 228.75 for the MSSM spectrum.

\section{EFFECTS OF R-SYMMETRY VIOLATION}

One would expect the explicit violation of $\mathrm{U}(1)_{R}$ in the superpotential to have important consequences for phenomenology and cosmology. The $Z_{2}$ subgroup of $\mathrm{U}(1)_{R}$, which is left unbroken by the soft SUSY-breaking terms, is called matter parity since all the matter (quark and lepton) superfields change sign under it. Combined with the $Z_{2}$ fermion parity (under which all fermions change sign) yields R-parity, which, if unbroken, guarantees the stability of the LSP. In our model, however, matter parity is violated along with the $\mathrm{U}(1)_{R}$ by the two explicitly displayed terms in the last line of the RHS of Eq. (1), which are needed for generating an acceptable BAU. Thus, Rparity is broken and, consequently, the LSP generically becomes unstable and decays rapidly, rendering it unsuitable for the role of dark matter and leading to distinctive collider signatures.

In our case, if the LSP contains a Higgsino component, it could decay into a pair of electroweak Higgs bosons and a lepton. The dominant diagrams are constructed from the $\mathrm{U}(1)_{R}$ and R-parity violating Yukawa vertices $\zeta_{j}\left(h_{1} h_{2}\right) \nu_{j}^{c}$ or $\zeta_{i}^{\prime} h_{1} \epsilon T l_{i}$ with the fermionic $\nu_{j}^{c}$ or $T$ connected to the fermionic $\nu_{j}^{c}$ or $\bar{T}$ of the Yukawa couplings $h_{\nu i j}\left(h_{2} l_{i}\right) \nu_{j}^{c}$ or $h_{\bar{T}} h_{2} \epsilon \bar{T} h_{2}$ respectively via a mass insertion. For the numerical values of the parameters that we consider (see Secs. VI]andVII), we find that the LSP lifetime can be as low as about $10^{-1}$ sec. However, it is easy to block kinematically the LSP decay by taking its mass to be smaller than twice the mass of the lightest Higgs boson, which is very reasonable. Thus, it is perfectly possible to rescue the LSP as dark matter candidate.

Our model could also predict the existence of other $\mathrm{R}$-parity violating processes at low energies besides the LSP decay. The R-parity breaking superpotential couplings involve at least one superheavy field (a RHN or $\mathrm{SU}(2)_{L}$ triplet). On integrating out these heavy fields, one generally obtains effective R-parity violating operators involving only MSSM fields. These operators, if they have dimension five or higher, do not lead to detectable processes since they are suppressed by some powers of $M_{T}$ or $M_{\nu_{i}^{c}}$. However, dimension four operators such as the effective scalar vertices $h_{1} h_{2} h_{2}^{*} \tilde{l}_{i}^{*}$ or $h_{1} \tilde{l}_{i} \tilde{l}_{j}^{*} \tilde{l}_{k}^{*}$, which originate from the superpotential couplings $\zeta_{j}\left(h_{1} h_{2}\right) \nu_{j}^{c}$, $h_{\nu i j}\left(h_{2} l_{i}\right) \nu_{j}^{c}$ or $\zeta_{i}^{\prime} h_{1} \epsilon T l_{i}, h_{T j k} l_{j} \epsilon T l_{k}$ respectively, can lead 
to low-energy R-parity violating processes which may be detectable in the future colliders (see Sec. VII).

It is well known 33 that, in any leptogenesis scenario with RHNs or $\mathrm{SU}(2)_{L}$ triplets, it is important to ensure that the primordial lepton asymmetry is not erased by lepton number violating $2 \rightarrow 2$ scattering processes such as $l_{i} \tilde{l}_{j} \rightarrow h_{2}^{*} \tilde{\tilde{h}}_{2}$ or $l_{i} \tilde{h}_{2} \rightarrow h_{2}^{*} \tilde{l}_{j}^{*}$ at all temperatures between $T_{\mathrm{rh}}$ and about $100 \mathrm{GeV}$. In our model, due to the presence of the R-parity violating superpotential couplings, there exist some extra processes of this type such as $\tilde{h}_{1} \tilde{l}_{j} \rightarrow h_{2}^{*} \tilde{\bar{h}}_{2}$ or $\tilde{h}_{1} \tilde{h}_{2} \rightarrow h_{2}^{*} \tilde{l}_{j}^{*}$, which are derived from diagrams similar to the ones mentioned above for the LSP decay. In addition to all these processes which correspond to effective operators of dimension five (or higher), we also have dimension four R-parity (and lepton number) violating processes such as $h_{1} h_{2} \rightarrow h_{2} \tilde{l}_{i}$ or $h_{1} \tilde{l}_{i} \rightarrow \tilde{l}_{j} \tilde{l}_{k}$, which are derived from the effective four-scalar vertices in the previous paragraph.

The initial lepton asymmetry is protected [32] by SUSY at temperatures between $T_{\text {rh }}$ and about $10^{7} \mathrm{GeV}$. For $T \lesssim 10^{7} \mathrm{GeV}$, one can show that all the lepton number violating $2 \rightarrow 2$ scattering processes which result from effective operators of dimension five or higher are well out of equilibrium for the values of the parameters used here. The dimension four lepton number violating processes, however, are generally in equilibrium and, thus, special care is needed in order to retain the initial lepton asymmetry in our model. We will return to this issue in the next section.

As already explained, the classical flatness of the inflationary path in the limit of global SUSY is ensured, in our model, by a continuous R-symmetry enforcing a linear dependence of the superpotential on $S$. This is retained 22 even after SUGRA corrections, given a reasonable assumption about the Kähler potential. The solution [2] to the $\mu$ problem is also reliant on the R-symmetry. These aspects of the model are not affected by the explicit Rsymmetry breaking we consider.

In the model, some R-symmetry violating couplings are present in the superpotential and some not. Thanks to the nonrenormalization property of SUSY, this situation is stable under radiative corrections, but one may consider it unnatural since there is no symmetry to forbid the terms we set to zero.

\section{NEUTRINO MASSES AND PRESERVATION OF LEPTON ASYMMETRY}

The two distinct sources of neutrino masses in the model yield the following mass matrix of light neutrinos:

$$
m_{\nu i j}=-\left\langle h_{2}\right\rangle^{2}\left[\frac{h_{T i j}}{h_{\bar{T}} M_{T}}+h_{\nu i k} \frac{1}{M_{\nu_{k}^{c}}} h_{\nu j k}\right] .
$$

The terms in this expression are generally complex with physical relative phases between the two contributions.
Comparing this to the combination of parameters appearing in the lepton asymmetry per inflaton decay, we see that there is no obvious correlation between the two. Hence, we have here more freedom to choose values for the parameters than in the case of leptogenesis with a single source of neutrino mass. However, this freedom is reduced by requiring that the lepton asymmetry is preserved until the electroweak phase transition.

As discussed in Sec. $\mathbb{V}$ there exist unsuppressed dimension four R-parity and lepton number violating $2 \rightarrow 2$ scattering processes which can erase the primordial lepton asymmetry. They originate from the effective fourscalar operators $h_{1} h_{2} h_{2}^{*} \tilde{l}_{i}^{*}$ or $h_{1} \tilde{l}_{i} \tilde{l}_{j}^{*} \tilde{l}_{k}^{*}$ with coupling constants $\sum_{j} \zeta_{j} h_{\nu i j}^{*}$ or $\zeta_{i}^{\prime} h_{T j k}^{*}$ respectively. The former operator violates, in particular, the $i$-lepton number $\left(L_{i}\right)$, i.e. the part of the total lepton number which corresponds to the $i$ th fermion family. Therefore, in order to retain at least one part of the lepton asymmetry, we need to impose the condition that

$$
\sum_{j} \zeta_{j} h_{\nu i j}^{*}=0
$$

for some value of $i$ [34]. To generate a $L_{i}$ asymmetry, we need to take a nonzero $\zeta_{i}^{\prime}$, as it is obvious from Eqs. (2) and (6)-(8). The operators $h_{1} \tilde{l}_{i} \tilde{l}_{j}^{*} \tilde{l}_{k}^{*}$ with $j, k \neq i$ or $j=$ $k=i$ will then violate $L_{i}$, thereby leading to the erasure of the primordial $L_{i}$ asymmetry. To avoid this, we must take $h_{T j k}$ to vanish, unless exactly one of the indices is equal to $i$, i.e. we allow only $h_{T i k}=h_{T k i} \neq 0$ for $k \neq i$. Moreover, if $h_{T i k} \neq 0$ then we require $\zeta_{j}^{\prime}=0$ for $j \neq i$ to avoid generating the $L_{i}$ violating operator $h_{1} \tilde{l}_{j} \tilde{l}_{i}^{*} \tilde{l}_{k}^{*}$.

We must further ensure that there are no processes in equilibrium which violate $L_{i}$, while conserving $L$. Such processes result from the four-scalar operators $h_{2} \tilde{l}_{k} h_{2}^{*} \tilde{l}_{i}^{*}$ $(k \neq i)$ which originate from the superpotential coupling $h_{\nu k j}\left(h_{2} l_{k}\right) \nu_{j}^{c}$. Thus, we must take

$$
\sum_{j} h_{\nu k j} h_{\nu i j}^{*}=0
$$

for the value of $i$ which satisfies Eq. (12) and all values of $k$ which are different from this $i$. Also, the operators $h_{1} \tilde{l}_{i} h_{1}^{*} l_{j}^{*}$ with $j \neq i$, which originate from the superpotential coupling $\zeta_{j}^{\prime} h_{1} \epsilon T l_{j}$, violate $L_{i}$, but not $L$. So, we must take $\zeta_{j}^{\prime}=0$ for $j \neq i$. Finally, the operators $\tilde{l}_{j} \tilde{l}_{k} \tilde{l}_{m}^{*} \tilde{l}_{n}^{*}$ derived from the superpotential term $h_{T j k} l_{j} \epsilon T l_{k}$ could violate $L_{i}$. However, as explained above, $h_{T j k}$ should vanish unless exactly one index is equal to $i$. Thus, the remaining operators automatically conserve $L_{i}$. In summary, for an asymmetry to be generated in $L_{i}$ and not wiped out by scattering processes, we require that $\zeta_{i}^{\prime} \neq 0$ and $\zeta_{j}^{\prime}=0$ for all $j \neq i$. Also, $h_{T j k}=0$ unless exactly one of $j$ or $k$ is equal to $i$ and Eqs. (12) and (13) should hold for all $k \neq i$.

We take the neutrino mass ordering $m_{1}<m_{2}<m_{3}$ and adopt the normal hierarchical scheme of neutrino masses, where the solar and atmospheric neutrino mass ${ }^{2}$ 
differences are identified with $\delta m_{21}^{2}$ and $\delta m_{31}^{2}$ respectively. Analysis [35] of the CHOOZ experiment [36] shows that the mixing angle $\theta_{13}$ can be taken equal to zero. Moreover, the fact that $\delta m_{21}^{2} \ll \delta m_{31}^{2}$ implies that, when considering atmospheric neutrino oscillations, it is a good approximation to set $\delta m_{21}^{2}=0$. For simplicity, we further take $\alpha=\beta$, where $\alpha, \beta$ are the Majorana phases in the leptonic mixing matrix associated with the two lighter neutrino mass eigenstates $\hat{\nu}_{1}, \hat{\nu}_{2}$ respectively.

Under these circumstances, the weak interaction eigenstate $\nu_{1}$ decouples from the other two $\nu_{2}, \nu_{3}$ in the neutrino mass matrix. A simple choice of parameters which leads to this decoupling is $h_{\nu 1 j}=0$ for $j=2,3, h_{\nu i 1}=0$ for $i=2,3$ and $h_{T 11}=h_{T 1 j}=h_{T j 1}=0$ for $j=2,3$. Eq. (13) is then automatically satisfied for $k=1$ and $i=2,3$, which means that processes corresponding to the operators $h_{2} \tilde{l}_{1} h_{2}^{*} \tilde{l}_{i}^{*}(i=2,3)$ which violate $L_{1}$, while conserving $L$, are avoided. If we further take $\zeta_{1}^{\prime}=0$, $L_{1}$ violating and $L$ conserving processes from the operators $h_{1} \tilde{l}_{1} h_{1}^{*} \tilde{l}_{j}^{*}(j=2,3)$ are also absent. Moreover, our choice of $h_{T j k}$ ensures that no such processes from operators of the type $\tilde{l}_{j} \tilde{l}_{k} \tilde{l}_{m}^{*} \tilde{l}_{n}^{*}$ appear. Eq. (12) with $i=1$ $(i=2,3)$ receives contribution only from the term(s) with $j=1(j=2,3)$. Also, $L$ and $L_{1}$ violating processes from the operators $h_{1} \tilde{l}_{i} \tilde{l}_{j}^{*} l_{k}^{*}$ do not exist for our choice of parameters. Note that there are no $L_{1}$ violating processes in equilibrium from the superpotential terms $\zeta_{i}^{\prime} h_{1} \epsilon T l_{i}, h_{T j k} l_{j} \epsilon T l_{k}$. The only possible $L_{1}$ violating processes come from the operator $h_{1} h_{2} h_{2}^{*} \tilde{l}_{1}^{*}$, which is though $L_{2}$ and $L_{3}$ conserving. The fact that $\zeta_{1}^{\prime}=0$ implies that no primordial $L_{1}$ asymmetry is generated as one can see from Eqs. (2) and (6)-(8). Also, the $L_{2}$ and $L_{3}$ asymmetries cannot turn into $L_{1}$ asymmetry by $2 \rightarrow 2$ scattering processes since, as explained, there are no such processes in equilibrium which simultaneously violate $L_{1}$ and $L_{2}$ or $L_{3}$. All these facts allow us to ignore the first family and concentrate on the two heaviest families.

From the recent global analysis [37] of neutrino oscillation data, we take the best-fit value of the atmospheric neutrino mass $^{2}$ difference which, in the case of the normal hierarchical scheme, yields the value $m_{3} \simeq 5.1 \times 10^{-2} \mathrm{eV}$ for the heaviest neutrino mass. Also, the mixing angle $\theta_{23}$ is identified with its best-fit value which is about $\pi / 4$ and $m_{2}$ is taken equal to zero consistently with the fact that we are considering only atmospheric neutrino oscillations. So, the neutrino mass matrix elements in Eq. (11) must satisfy the following restrictions:

$$
m_{\nu 22}=m_{\nu 33}=m_{\nu 23}=\frac{1}{2} m_{3} .
$$

(Note that, in the case of three neutrino flavors, our choice of parameters supplemented, for consistency, with a vanishing $m_{1}$ yields the extra restriction $h_{\nu 11}=0$.) These equalities need not be exact, however, we will take them as so for simplicity. We choose to maintain the $L_{3}$ asymmetry, which requires that Eq. (12) holds for $i=3$ and Eq. (13) for $k=2, i=3$. So, we obtain the following extra conditions:

$$
\begin{gathered}
h_{\nu 32}^{*}+R h_{\nu 33}^{*}=0, \\
h_{\nu 22} h_{\nu 32}^{*}+h_{\nu 23} h_{\nu 33}^{*}=0,
\end{gathered}
$$

where $R=\zeta_{3} / \zeta_{2}$. Applying the results of the previous discussion, we require $\zeta_{3}^{\prime}$ to be nonzero and $\zeta_{2}^{\prime}=0$ (so no $L_{2}$ asymmetry is generated); we have also $h_{T 22}=$ $h_{T 33}=0$, so the only nonzero coupling constant of the type $h_{T j k}$ is $h_{T 23}=h_{T 32}$. Given the value of the right handed neutrino masses, $M_{T}$ and $h_{\bar{T}}$, the five conditions in Eqs. (14) and (15) can be solved, using the mass formula in Eq. (11), to find a relation between the complex Yukawa coupling constants $h_{i j}(i, j=2,3)$ and the complex parameters $R$ and $h_{T 23}$.

Note that the presence of $L$ and $B-L$ violating $2 \rightarrow 2$ scattering processes leads to a moderate modification of the numerical factors in Eq. (9). However, this modification depends on details and we will, thus, ignore it.

\section{NUMERICAL RESULTS}

We saturate the gravitino bound on the reheat temperature by taking $T_{\mathrm{rh}}=10^{10} \mathrm{GeV}$ which is acceptable [10] provided that the branching ratio of the gravitino decay to photons and photinos is less than unity. We also fix the parameter $\kappa$ to the value $10^{-4}$. The cosmic microwave background explorer (COBE) value of the quadrupole anisotropy of the $\mathrm{CMBR}\left((\delta T / T)_{Q} \simeq 6.6 \times 10^{-6}\right)[38]$ is then reproduced for $\lambda \simeq 1.44 \times 10^{-4}(>\kappa$ as it should $)$ and $M \simeq 3.53 \times 10^{15} \mathrm{GeV}$. Thus, $m_{\mathrm{inf}} \simeq 4.99 \times 10^{11} \mathrm{GeV}$. The spectral index of density perturbations comes out practically equal to unity.

We now wish to demonstrate that our leptogenesis mechanism can easily reproduce the best-fit value of the $\mathrm{BAU}, n_{B} / s \simeq 8.66 \times 10^{-11}$, derived from the recent WMAP data [ [] ] with a natural choice of parameters. For simplicity, we approximate all the right handed neutrino masses to the same value $M_{\nu^{c}}$. (Note that we do not make use of resonance effects for degenerate heavy neutrinos, so this approximation will not strongly influence our results.) Eq. (15) implies that $h_{\nu 32}=-R^{*} h_{\nu 33}$ and $h_{\nu 23}=R h_{\nu 22}$. (Note that $h_{\nu 33} \neq 0$ for a nonvanishing $L_{3}$ asymmetry to be produced.) Substituting these in Eq. (14), we then obtain

$$
\begin{aligned}
& h_{\nu 22}= \pm i\left(\frac{m_{3} M_{\nu^{c}}}{2\left\langle h_{2}\right\rangle^{2}}\right)^{\frac{1}{2}} \frac{1}{\left(1+R^{2}\right)^{\frac{1}{2}}}, \\
& h_{\nu 33}= \pm i\left(\frac{m_{3} M_{\nu^{c}}}{2\left\langle h_{2}\right\rangle^{2}}\right)^{\frac{1}{2}} \frac{1}{\left(1+R^{* 2}\right)^{\frac{1}{2}}},
\end{aligned}
$$

and

$$
\pm \frac{R-R^{*}}{\left(1+R^{2}\right)^{\frac{1}{2}}\left(1+R^{* 2}\right)^{\frac{1}{2}}}=1+\frac{2 h_{T 23}\left\langle h_{2}\right\rangle^{2}}{h_{\bar{T}} m_{3} M_{T}} .
$$


Note that the signs of $h_{\nu 22}$ and $h_{\nu 33}$ can be chosen independently; however, the sign on the left hand side (LHS) of Eq. (18) is the product of the two.

The LHS of Eq. (18) is imaginary. Therefore, the real part of the RHS of this equation must vanish, which gives

$$
\operatorname{Re} h_{T 23}=-\frac{h_{\bar{T}} m_{3} M_{T}}{2\left\langle h_{2}\right\rangle^{2}} .
$$

This implies that

$$
\frac{R-R^{*}}{\left(1+R^{2}\right)^{\frac{1}{2}}\left(1+R^{* 2}\right)^{\frac{1}{2}}}=i \frac{2 \operatorname{Im}\left(h_{T 23}\right)\left\langle h_{2}\right\rangle^{2}}{h_{\bar{T}} m_{3} M_{T}} \equiv i \xi,
$$

which yields

$$
\xi^{2}|R|^{4}+2\left[\left(1+\xi^{2}\right) \cos 2 \varphi-1\right]|R|^{2}+\xi^{2}=0,
$$

where $\varphi$ is the phase of the complex parameter $R$. This equation admits real solutions for $|R|^{2}$ provided that $-1 \leq \cos 2 \varphi \leq\left(1-\xi^{2}\right) /\left(1+\xi^{2}\right)$. They turn out to be nonnegative and are given by

$$
\begin{aligned}
|R|^{2}= & \xi^{-2}\left\{\left[1-\left(1+\xi^{2}\right) \cos 2 \varphi\right] \pm\left(1+\xi^{2}\right)^{\frac{1}{2}}\right. \\
& {\left.\left[\left(1+\xi^{2}\right) \cos ^{2} 2 \varphi-2 \cos 2 \varphi+\left(1-\xi^{2}\right)\right]^{\frac{1}{2}}\right\} . }
\end{aligned}
$$

From Eqs. (2) and (6)-(9), we see that, in our case, the baryon asymmetry is proportional to $\zeta_{3}^{\prime} h_{\bar{T}}\left|\zeta_{2} h_{\nu 33} \operatorname{Im} R\right|$ for given $M_{\nu^{c}}, M_{T} \geq m_{\text {inf }} / 2 \simeq 2.5 \times 10^{11} \mathrm{GeV}$. Using Eqs. (17) and (20), we can further show that this expression is proportional to $\zeta_{3}^{\prime} h_{\bar{T}}\left|\zeta_{2} \xi^{1 / 2} R^{1 / 2}\right|(1-\cos 2 \varphi)^{1 / 4}$. As discussed in Sec. III the parameters $\zeta_{3}^{\prime},\left|\zeta_{2}\right|$ and $\left|\zeta_{3}\right|=\left|R \| \zeta_{2}\right|$ should not exceed $M / M_{S} \simeq 7 \times 10^{-3}$. To maximize $n_{B} / s$, we saturate this limit on $\zeta_{3}^{\prime}$ and on $\left|\zeta_{2}\right|$ or $\left|\zeta_{3}\right|$ for $|R| \leq 1$ or $|R|>1$ respectively.

In the former case, the baryon asymmetry, which is $\propto h_{\bar{T}}|\xi|^{1 / 2}|R|^{1 / 2}(1-\cos 2 \varphi)^{1 / 4}$, can be further maximized by choosing the positive sign in the RHS of Eq. (22) and taking $\cos 2 \varphi=-1$. We then get $|R|=\left(1+\xi^{-2}\right)^{1 / 2}+$ $|\xi|^{-1}$, which is always $>1$. Our requirement that $|R|$ does not exceed unity is fulfilled only asymptotically, i.e. for $|\xi| \rightarrow \infty$, where $|R| \rightarrow 1$. The baryon asymmetry is then $\propto h_{\bar{T}}|\xi|^{1 / 2}$ and is maximized by maximizing $h_{\bar{T}}$ and $\left|\operatorname{Im} h_{T 23}\right|$. It would thus be desirable to put both these quantities equal to unity. The parameter $h_{\bar{T}}$ can be readily fixed to unity and this improves the naturalness of our scheme. However, if $\left|\operatorname{Im} h_{T 23}\right|$ is too large, some moderate cancellations between different contributions to the neutrino mass matrix are required. So, we take values of $\left|\operatorname{Im} h_{T 23}\right|$ which are smaller than unity, but greater than, say, $10^{-2}$ to be consistent with the requirement that $|\xi| \gg 1$ for $M_{T}$ not much bigger than $m_{\text {inf }} / 2$.

In the case where $|R|>1$, we should saturate the limit on $\left|\zeta_{3}\right|$ rather than the limit on $\left|\zeta_{2}\right|$, which yields that the baryon asymmetry is $\propto h_{\bar{T}}|\xi|^{1 / 2}|R|^{-1 / 2}(1-\cos 2 \varphi)^{1 / 4}$. This is maximized by choosing the negative sign in the RHS of Eq. (22) and taking again $\cos 2 \varphi=-1$, which gives $|R|=\left(1+\xi^{-2}\right)^{1 / 2}-|\xi|^{-1}<1$. So, marginal consistency is achieved again for $|\xi| \gg 1$, where $|R| \rightarrow 1$. The

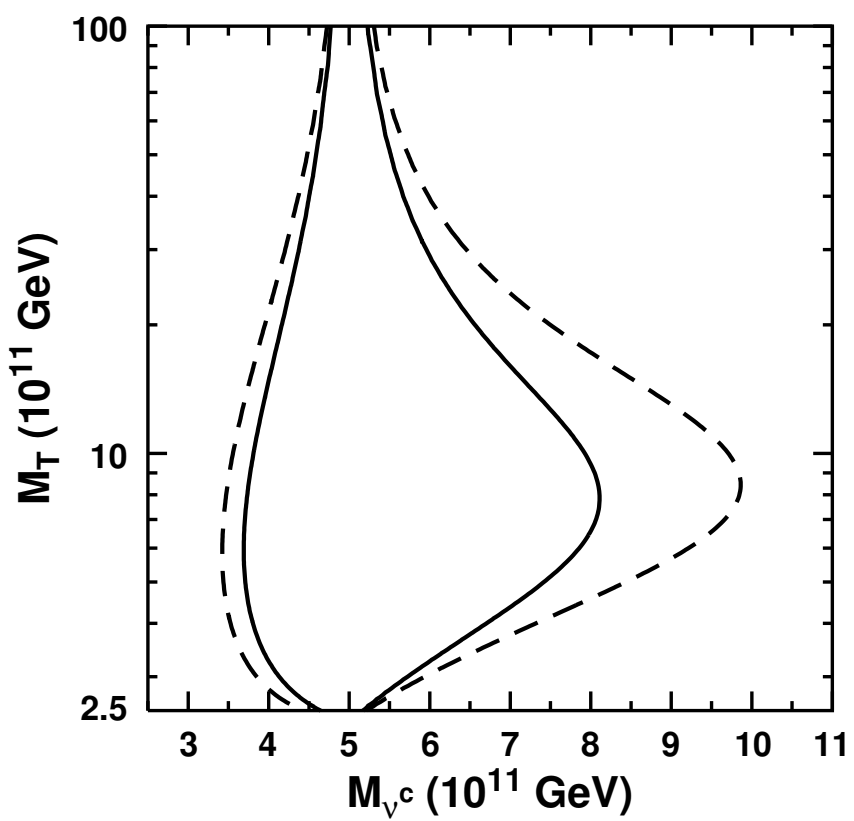

FIG. 3: The solutions of $n_{B} / s=8.66 \times 10^{-11}$ in the $M_{\nu^{c}}-M_{T}$ plane for $\operatorname{Im} h_{T 23}=0.02$ (solid lines) or 0.04 (dashed lines). The values of all the other parameters are given in the text.

baryon asymmetry is again $\propto h_{\bar{T}}|\xi|^{1 / 2}$ and is maximized by taking $h_{\bar{T}}=\left|\operatorname{Im} h_{T 23}\right|=1$. As in the previous case, we take $h_{\bar{T}}=1$ and $\left|\operatorname{Im} h_{T 23}\right| \sim 10^{-2}$. The result is obviously the same in both cases to a good approximation. The phase of $\zeta_{2}$ is appropriately adjusted in each case so that we obtain the maximal baryon asymmetry.

For each $M_{\nu^{c}}, M_{T} \geq m_{\text {inf }} / 2$, we calculate the BAU using Eqs. (6)-(9) with $\operatorname{Im} h_{T 23}=0.02$ or 0.04 and values for the other parameters as explained above. We then compare the result with the best-fit value of the BAU from WMAP. The resulting solutions in the $M_{\nu^{c}}-M_{T}$ plane are shown in Fig. 3 with the solid or dashed lines corresponding to $\operatorname{Im} h_{T 23}=0.02$ or 0.04 respectively. Note that there are two branches in each case, one with $M_{\nu^{c}}>m_{\text {inf }}$ and one with $M_{\nu^{c}}<m_{\text {inf }}$. They correspond to the two possible signs of the denominator in the RHS of Eq. (8) for $\epsilon_{V S}$. Actually, this quantity, as it involves a s-channel exchange of a $\nu^{c}$ boson, can be easily enhanced by letting $m_{\text {inf }}$ approach the $\nu^{c}$-pole. This fact assists us to achieve the WMAP value of $n_{B} / s$ with natural values of the parameters. It is important though to stress that there is no need to get too near the $\nu^{c}$-pole for reasonable values of $M_{T}$. In fact, the solution is very far from being unnaturally close to this pole.

As mentioned in Sec. V the explicit R-parity violation in our model, which is required for leptogenesis, has some low-energy signatures which come from dimension four effective scalar vertices and may be observable in the future colliders. Such signatures may typically be the three-body slepton decay processes

$$
\tilde{l}_{2} \rightarrow h_{1} h_{2} h_{2}^{*} \text { and } \tilde{l}_{2} \rightarrow h_{1} \tilde{l}_{3} \tilde{l}_{3}^{*},
$$


which can easily be kinematically allowed. (Note that, for our choice of parameters, similar $\tilde{l}_{1}$ decay processes do not appear, although they may be present in the general case.) The effective coupling constants of the processes in Eq. (23) are $\zeta_{2} h_{\nu 22}^{*}+\zeta_{3} h_{\nu 23}^{*}$ and $\zeta_{3}^{\prime} h_{T 23}^{*}$ respectively. Using the relation $h_{\nu 23}=R h_{\nu 22}$ and substituting $h_{\nu 22}$ from Eq. (16), we can easily show that the magnitude of the former coupling constant becomes $\simeq\left|\zeta_{2}\right|\left|2 \operatorname{Im} h_{T 23}\right|^{1 / 2}\left(M_{\nu^{c}} / M_{T}\right)^{1 / 2}$ in the large $|\xi|$ limit. For $\operatorname{Im} h_{T 23}=0.02$ and taking $M_{\nu^{c}} \simeq M_{T}$, which holds near the right corner of the solid line in Fig. 3] we find that the magnitude of the effective coupling constants of the processes in Eq. (23) is about $1.4 \times 10^{-3}$ and $1.4 \times 10^{-4}$ respectively. The corresponding decay rates are then of order $10^{-8} \mathrm{GeV}$ and $10^{-10} \mathrm{GeV}$ respectively for mass of the decaying slepton $\sim 1 \mathrm{TeV}$ and assuming that there is an appreciable gap between this mass and the sum of the masses of the decay products.

It is finally interesting to point out that, as one can readily show, our scheme fails to provide any useful predictions for the so far undetermined or not so accurately determined parameters in the neutrino mixing matrix, i.e. the three $\mathrm{CP}$-violating phases and the mixing angle $\theta_{13}$. The reason is that the number of parameters is such that successful leptogenesis is possible whatever the values of the complex phases and $\theta_{13}$.

\section{CONCLUSIONS}

We proposed a scenario of nonthermal leptogenesis following supersymmetric hybrid inflation, in the case where the decay of the inflaton to both heavy right handed neutrino and $\mathrm{SU}(2)_{L}$ triplet superfields is kinematically blocked. The primordial lepton asymmetry is generated through the direct decay of the inflaton into light particles. We implemented our scenario in the context of a simple SUSY GUT model which incorporates the standard version of SUSY hybrid inflation. The $\mu$ problem is solved via a $\mathrm{U}(1) \mathrm{R}$-symmetry which forbids the existence of an explicit $\mu$ term, while allows a trilinear superpotential coupling of the gauge singlet inflaton to the electroweak Higgs superfields. After the spontaneous break- ing of the GUT gauge symmetry, this singlet inflaton acquires a suppressed VEV due to the soft SUSY-breaking terms. Its trilinear coupling to the Higgs superfields then yields a $\mu$ term of the right magnitude.

The main decay mode of the inflaton is to a pair of electroweak Higgs superfields via the same trilinear coupling. The initial lepton asymmetry is created in the subdominant decay of the inflaton to a lepton and an electroweak Higgs superfield via the interference of one-loop diagrams with right handed neutrino and $\mathrm{SU}(2)_{L}$ triplet exchange respectively. The existence of these diagrams requires the presence of some specific superpotential couplings which explicitly violate the U(1) R-symmetry and R-parity. However, the broken R-parity need not have currently observable low-energy signatures, although it may have signatures detectable in future colliders. Also, the LSP can be made stable and, thus, be a possible candidate for cold dark matter.

In our analysis, we took into account the constraints from neutrino masses and mixing. There exist, in our model, two separate contributions to the neutrino mass matrix, which originate from the usual seesaw mechanism and from the $\mathrm{SU}(2)_{L}$ triplet superfields. The constraints arising from neutrino masses and mixing alone are not very stringent. However, the requirement that the primordial lepton asymmetry not be erased by lepton number violating processes before the electroweak phase transition is a much more stringent constraint on the parameters of the theory. Taking into account these constraints we found that the best-fit value of the BAU from the recent WMAP data can be easily achieved with natural values of parameters.

\section{Acknowledgements}

We thank A. Pilaftsis and K. Tobe for useful discussions and T. Hahn for his help with the software packages of Ref. [30]. T. D. thanks the Michigan Centre for Theoretical Physics for hospitality and a pleasant environment during their Baryogenesis Workshop. This work was supported by the European Union under RTN contracts HPRN-CT-2000-00148 and HPRN-CT-2000-00152.
[1] For a review see e.g. S.P. Martin, hep-ph/9709356

[2] G.R. Dvali, G. Lazarides and Q. Shafi, Phys. Lett. B 424, 259 (1998).

[3] T. Yanagida, in Proceedings of the Workshop on Unified Theories and Baryon Number in the Universe, edited by A. Sawada and A. Sugamoto (KEK Rep. No. 79-18, Tsukuba, Japan, 1979), p. 95; S.L. Glashow, in Quarks and Leptons, Cargése 1979, edited by M. Lévy et al. (Plenum, New York, 1980), p. 707; M. Gell-Mann, P. Ramond and R. Slansky, in Supergravity, edited by P. Van Nieuwenhuizen and D.Z. Freedman (North Holland, Amsterdam, 1979), p. 315; R.N. Mohapatra and G. Sen- janovic, Phys. Rev. Lett. 44, 912 (1980).

[4] G. Lazarides, Q. Shafi and C. Wetterich, Nucl. Phys. B181, 287 (1981); R.N. Mohapatra and G. Senjanović, Phys. Rev. D 23, 165 (1981); C. Wetterich, Nucl. Phys. B187, 343 (1981).

[5] A.R. Liddle and D.H. Lyth, Cosmological inflation and large-scale structure (Cambridge University Press, Cambridge, 2001).

[6] D.N. Spergel et al., Astrophys. J. Suppl. 148, 175 (2003); H.V. Peiris et al., ibid. 148, 213 (2003).

[7] M. Fukugita and T. Yanagida, Phys. Lett. B 174, 45 (1986). 
[8] P.J. O'Donnell and U. Sarkar, Phys. Rev. D 49, 2118 (1994); E. Ma and U. Sarkar, Phys. Rev. Lett. 80, 5716 (1998).

[9] M.Yu. Khlopov and A.D. Linde, Phys. Lett. B 138, 265 (1984); J. Ellis, J.E. Kim and D. Nanopoulos, Phys. Lett. B 145, 181 (1984); I.V. Falomkin, D.B. Pontecorvo, M.G. Sapozhnikov, M.Yu. Khlopov, F. Balestra and G. Piragino, Sov. J. Nucl. Phys. 39, 626 (1984).

[10] J.R. Ellis, D.V. Nanopoulos and S. Sarkar, Nucl. Phys. B259, 175 (1985); J.R. Ellis, G.B. Gelmini, J.L. Lopez, D.V. Nanopoulos and S. Sarkar, ibid. B373, 399 (1992).

[11] A. Pilaftsis, Phys. Rev. D 56, 5431 (1997).

[12] T. Hambye, E. Ma and U. Sarkar, Nucl. Phys. B602, 23 (2001); J.R. Ellis, M. Raidal and T. Yanagida, Phys. Lett. B 546, 228 (2002); A. Pilaftsis and T.E.J. Underwood, hep-ph/0309342

[13] G. Lazarides and Q. Shafi, Phys. Lett. B 258, 305 (1991).

[14] G. Lazarides and Q. Shafi, Phys. Rev. D 58, 071702 (1998).

[15] G. Lazarides, Phys. Lett. B 452, 227 (1999); hep-ph/99 05450.

[16] R. Allahverdi and A. Mazumdar, Phys. Rev. D 67, 023509 (2003).

[17] J.R. Ellis, M. Raidal and T. Yanagida, hep-ph/0303242

[18] E.J. Copeland, A.R. Liddle, D.H. Lyth, E.D. Stewart and D. Wands, Phys. Rev. D 49, 6410 (1994).

[19] G.R. Dvali, Q. Shafi and R.K. Schaefer, Phys. Rev. Lett. 73, 1886 (1994).

[20] A.D. Linde, Phys. Lett. B 259, 38 (1991); Phys. Rev. D 49, 748 (1994).

[21] R. Jeannerot, S. Khalil and G. Lazarides, hep-ph/01 06035.

[22] G. Lazarides, R.K. Schaefer and Q. Shafi, Phys. Rev. D
56, 1324 (1997).

[23] R. Jeannerot, S. Khalil and G. Lazarides, J. High Energy Phys. 07, 069 (2002).

[24] T. Hambye and G. Senjanović, hep-ph/0307237

[25] G. Lazarides, hep-ph/9904502 Lect. Notes Phys. 592, 351 (2002); hep-ph/0204294

[26] G. Lazarides and N.D. Vlachos, Phys. Lett. B 441, 46 (1998).

[27] G. Lazarides, hep-ph/9904372

[28] L. Covi, E. Roulet and F. Vissani, Phys. Rev. D 384, 169 (1996).

[29] B.A. Kniehl and A. Pilaftsis, Nucl. Phys. B474, 28 (1996); A. Pilaftsis, ibid. B504, 61 (1997).

[30] T. Hahn, Acta Phys. Polon. B 30, 3469 (1999).

[31] L.G. Cabral-Rosetti and M.A. Sanchis-Lozano, hep-ph/0206081

[32] L.E. Ibáñez and F. Quevedo, Phys. Lett. B 283, 261 (1992).

[33] J.A. Harvey and M.S. Turner, Phys. Rev. D 42, 3344 (1990).

[34] It is not necessary for this equation, or the other constraints on $L_{i}$ violating couplings, to be satisfied exactly: the dangerous dimension four operators will not come into equilibrium if their coupling constants are less than $10^{-7}$. However, we take them to vanish exactly in order to simplify the discussion.

[35] G.L. Fogli, G. Lettera, E. Lisi, A. Marrone, A. Palazzo and A. Rotunno, Phys. Rev. D 66, 093008 (2002).

[36] M. Apollonio et al., Phys. Lett. B 466, 415 (1999).

[37] G.L. Fogli, E. Lisi, A. Marrone and D. Montanino, Phys. Rev. D 67, 093006 (2003).

[38] C.L. Bennett et al., Astrophys. J. 464, L1 (1996). 